\title{
Skin lesions in children admitted to the paediatric intensive care unit: an observational study
}

\author{
J. Henk Sillevis Smitt • Job B. M. van Woensel • \\ Albert P. Bos
}

Received: 30 November 2010 / Accepted: 15 February 2011 /Published online: 2 March 2011

(C) The Author(s) 2011. This article is published with open access at Springerlink.com

\begin{abstract}
We analysed, by a prospective observational study over a 3-year period, the frequency and character of dermatological symptoms and diseases in children admitted to a tertiary general paediatric intensive care unit (PICU) of a university hospital. Skin problems were observed in 42 of 1,800 children admitted. There was a large variability in dermatological diseases in the PICU. In the majority (23/42), the skin problem was a dermatological manifestation of an underlying illness that caused admission to the PICU, e.g. infection, vasculitis or drug reaction. In four of the seven children who died, a direct relation between the dermatological disease and the cause of death could be established. Although the number of patients with relevant dermatological problems was limited, we believe that a skilled paediatric dermatologist is able to contribute to the effectiveness of diagnostic and therapeutic processes in paediatric intensive care patients.
\end{abstract}

Keywords Paediatric intensive care - Iatrogenic · Infection · Purpura

J. H. Sillevis Smitt $(\bowtie)$

Department of Dermatology, A0-228, Academic Medical Center, University of Amsterdam,

Postbox 22660, 1100 DD Amsterdam, The Netherlands

e-mail: j.h.sillevissmitt@amc.uva.nl

J. B. M. van Woensel

Department of Paediatric Intensive Care,

Academic Medical Center, Emma Children's Hospital,

University of Amsterdam,

Amsterdam, The Netherlands

\section{A. P. Bos}

Department of Paediatric Intensive Care,

Academic Medical Center, Emma Children's Hospital,

University of Amsterdam,

Amsterdam, The Netherlands

\section{Introduction}

Due to the character of paediatric intensive care having its focus on derailment and support of vital functions, dermatological symptoms are generally not a main issue during admission of children to a paediatric intensive care unit (PICU). The classical presentation of meningococcal disease is an excellent example to show that a dermatological symptom may contribute to the diagnosis of a life-threatening illness. On the other hand, the skin may be forgotten and skin lesions may be ignored in daily clinical examination [1] Besides, skin symptoms may develop during admission to the PICU. To our best knowledge, no reports on the incidence and character of skin lesions in patients admitted to the PICU exist.

In order to evaluate the frequency and character of skin symptoms occurring in paediatric intensive care patients, we performed a prospective study. The PICU of the Emma Children's Hospital/Academic Medical Center Amsterdam is a tertiary PICU with 14 beds, admitting 600 medical, surgical and trauma patients from the greater Amsterdam area annually.

\section{Methods}

The study was performed from February 1, 2002 through March 1, 2005. All children with abnormalities of the skin were analysed by a paediatric dermatologist who was assisted by residents receiving their training in paediatric dermatology. When the skin disease was not readily recognized and diagnosed, skin biopsies were taken for pathological analysis. The ethical committee was not contacted because of the observational nature of the study. 
Table 1 Number of children showing skin lesions, arranged according to morphology

\begin{tabular}{ll}
\hline Morphology & Number \\
\hline Purpuric lesions & $19^{\mathrm{a}}$ \\
Exanthema/erythema & $11^{\mathrm{a}}$ \\
Bullous eruption & 4 \\
Ulceration & $5^{\mathrm{b}}$ \\
Other & 6 \\
\hline
\end{tabular}

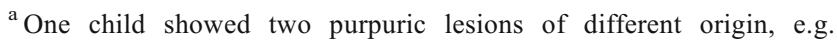
meningococcal disease and complication of indwelling line; one child showed purpura as complication of indwelling line and an exanthematous drug eruption

${ }^{\mathrm{b}}$ One child showed two ulcerative lesions of different origin, e.g. pressure ulcer and complication of indwelling arterial catheter

\section{Results}

A total of 42 children with skin problems out of 1,800 admitted to the PICU were seen, representing only $2.3 \%$ of all admissions to the PICU in the period under study. Twentythree were female, 19 were male; median age 30 months (range 0-216 months). Thirty-eight children showed one dermatological problem and four had two dermatological problems.

In Table 1, the number of children with skin lesions arranged according to their morphology is shown, and in Table 2, the skin lesions are arranged according to cause and diagnosis.
In seven patients, a skin biopsy was taken during their stay on the PICU. In the case of dystrophic epidermolysis bullosa, histopathological and molecular analysis of skin samples was already done in the past. In particular in drug eruptions, Henoch Schönlein vasculitis, toxic epidermal necrolysis (TEN) and necrotising fasciitis biopsies were helpful in making a definite diagnosis.

In four of the seven children with dermatological problems who died during their stay in the PICU, a direct relation between the dermatological disease and the cause of death could be established. Two patients died of severe progressive necrotising fasciitis (in one patient caused by Pseudomonas aeruginosa related to idiopathic congenital neutropenia and in another patient caused by pyogenic streptococci as complication of chickenpox with refractory circulatory failure). One patient died of circulatory failure due to neutropenic enterocolitis with TEN as a dermatological manifestation. The patient with dystrophic epidermolysis bullosa died of respiratory insufficiency due to pneumonia.

\section{Discussion}

In this study, we evaluated the frequency and character of dermatological symptoms occurring in patients admitted to the PICU. Skin problems did not seem to be a major item; in $2.3 \%$ of the admissions, the dermatologist was consulted. In view of the fact that we did find only a small cohort of
Table 2 Number of children showing skin lesions, arranged according to cause and diagnosis

\begin{tabular}{|c|c|c|}
\hline Cause & Diagnosis & Number \\
\hline \multirow[t]{6}{*}{ Iatrogenic } & Complications of indwelling lines & 8 \\
\hline & Drug eruption & 4 \\
\hline & Pressure ulcer & 2 \\
\hline & Complication tracheotomy & 2 \\
\hline & Friction blister (due to fixation intravenous line splint) & 1 \\
\hline & & Total 17 \\
\hline \multirow[t]{8}{*}{ Infectious } & Meningococcal sepsis/meningitis & 5 \\
\hline & Toxic shock syndrome & 4 \\
\hline & Necrotizing fasciitis & 2 \\
\hline & Complications varicella zoster infection & 3 \\
\hline & Staphylococcal scalded skin syndrome & 1 \\
\hline & Streptococcal meningitis & 1 \\
\hline & Others & 3 \\
\hline & & Total 19 \\
\hline \multirow[t]{6}{*}{ Others } & Henoch Schönlein vasculitis & 2 \\
\hline & Haemangioma & 2 \\
\hline & Haematoma & 4 \\
\hline & Toxic epidermal necrolysis (TEN) & 1 \\
\hline & Dystrophic epidermolysis bullosa & 1 \\
\hline & & Total 10 \\
\hline
\end{tabular}

${ }^{\text {a }}$ Three children are included in two groups, one child is included in two diseases of one group 
children with skin problems $(n=42)$, it is impossible to come to solid conclusions but trends can be observed. Since this is the first study to evaluate dermatological problems exclusively in paediatric ICU patients, our results are not comparable to other studies. One previous study reported dermatological problems in PICU patients only as part of a larger study (concerning 358 ICU consultations) including adults as well. Unfortunately, the total number of ICU admissions was not given [3]. They reported that 40 of 358 patients of the intensive care consultations were done in the paediatric ICU, but did not further specify the paediatric patients nor their diagnoses [3]. In another study, in adult patients only, the prevalence of dermatological problems in the ICU was much higher than we found, being more than $10 \%$ [1]. A retrospective study reported in 1995 including just adult patients over 14 consecutive months with a total of 1,194 admissions to the ICU recorded 27 patients with major skin diagnoses only [2]. Their numbers (27/1194) are comparable to ours (42/1800), but they were looking at major dermatological diseases only, while in our prospective study, we recorded every dermatological complaint.

Other studies concerning children in a PICU focussed on a single skin problem in particular, e.g. "preventable harm occurring to critically ill children" [4], catheter-related bloodstream infections [9], pressure ulcers $[5,7]$ or candida colonization in sepsis or septic shock [8].

Our results show that in the majority (23/42), the skin problem was a dermatological manifestation of the underlying illness that caused admission to the PICU, e.g. infection, vasculitis, drug reaction (Table 2). In several of these cases, dermatological expertise (including histopathological analysis) was helpful in establishing the final diagnosis. A substantial number (15/42) of dermatological problems found was related to the intensive care regimen (Table 2), such as some of the drug reactions, the pressure ulcers or the complications of indwelling catheters.

Compared to reported data of adults, the number of skin problems related to drug reactions seems to be lower in our study(5/42 in our study vs 77/358 [3]), while the frequency of skin problems of infectious origin seems to be somewhat higher (19/42 in our study vs 18/46 reported in adults[1]). It is well known that the incidence of admission to hospital for adverse drug reactions in general is increasing with age [6]. This suggests that age might be the cause of a low number of skin problems related to drugs in our study. However, no data concerning adverse drug reactions of the skin in particular, nor data of adverse skin reactions from adult ICUs are known. Comparing our data with published figures in adults, and as far as available in children, demonstrates also some other conspicuous differences. Pressure ulcers in PICU patients are reported to have an incidence between 7\% [5] and $11.7 \%$ [7] in children, while in our study, only two children $[2 / 1,800(0,1 \%)$ of the admissions in the PICU] showed a pressure ulcer over a 3-year period. We think this low incidence may be due to the fact that the PICU described is taking part in a hospital-wide pressure ulcer prevention program. In our study, no signs of candida colonization of the skin were found, but no routine cultures of the skin were taken. Singhi et al., [8] in case of severe sepsis or shock, did find skin colonization with candida by swabs in $34 \%$ of their PICU patients.

In conclusion, in this prospective study during a 3-year period, we found a considerable variation in dermatological diseases in the PICU (Table 2).

Conflicts of interest This research was not sponsored.

Open Access This article is distributed under the terms of the Creative Commons Attribution Noncommercial License which permits any noncommercial use, distribution, and reproduction in any medium, provided the original author(s) and source are credited.

\section{References}

1. Badia M, Trujillano J, Gascó E et al (1999) Skin lesions in the ICU. Int Care Med 25:1271-1276

2. Dunnill MGS, Handfield-Jones SE, Treacher D et al (1995) Dermatology in the intensive care unit. Brit J Dermatol 132:226-235

3. Fischer M, Soukop J, Wohlrab J et al (2004) Key dermatological symptoms in the intensive care unit. Int J Dermatol 43:780-782

4. Larsen GY, Donaldson AE, Parker HB et al (2007) Preventable harm occurring to critically ill children. Pediatr Crit Care Med 8:331-336

5. Pasek TA, Geyser A, Sidoni M et al (2008) Skin care team in the pediatric intensive care unit: a model for excellence. Crit Care Nurse 28:125-135

6. Pouyanne P, Haramburu F, Imbs JL et al (2000) Admission to hospital caused by adverse drug reactions: cross sectional incidence study. BMJ 320:1036

7. Schindler CA, Mikhailov TA, Fischer K et al (2007) Skin integrity in critically ill and injured children. Am J Crit Care 16:568-574

8. Singhi S, Rao R, Chakrabart A (2008) Candida colonization and candidemia in a pediatric intensive care unit. Pediatr Crit Care Med 9:91-95

9. Smith MJ (2008) Catheter-related bloodstream infections in children. Am J Infect Control 36:S173.e1-S173.e3 